\title{
Human immunodeficiency virus (HIV) infection for the general physician
}

\author{
R.J.C. Gilson and I.V.D. Weller
}

Academic Department of Genito Urinary Medicine, Middlesex Hospital Medical School, James Pringle House, Middlesex Hospital, London WIN 8AA, UK.

\section{Introduction}

The acquired immune deficiency syndrome (AIDS) is now recognized as the end stage of a disease caused by human immunodeficiency virus infection (HIV, formerly LAV/HTLV-III). The Centers for Disease Control (CDC) definition of AIDS only identifies those infected individuals with certain opportunistic infections, tumours or associated diseases who have developed a progressive irreversible immune deficiency. ${ }^{1}$ For every case of AIDS, it has been estimated that there are 30 to 300 individuals infected by the virus, who may exhibit acute or chronic features of the infection, or remain asymptomatic. ${ }^{2}$ The well recognized risk groups reflect the sexual, parenteral and perinatal routes of transmission of the virus. ${ }^{3,4,5}$

HIV is a double-stranded RNA retrovirus, which on penetration of a susceptible cell bearing a CD4 antigen receptor $(\mathrm{CD}=$ cluster differentiation), makes a DNA copy of itself. This process uses a viral reverse transcriptase enzyme. This proviral DNA is then incorporated into the host genetic material where it may remain latent or periodically produce new RNA copies. New viral particles are assembled from the RNA, core and envelope proteins. ${ }^{6}$ Isolates of HIV show considerable genetic heterogeneity, particularly in the envelope gene region. Recently a similar virus (LAV-2 or HIV-2) has been isolated from patients with AIDS from West Africa, and two Caucasian homosexual men in Paris. Its morphological and biological features are similar to HIV-1 but it differs in some of its antigenic components, notably the envelope glycoprotein. ${ }^{7,8}$ The primary defect induced by HIV infection is in cellular immunity. The CD4 antigen expressed by the helper/inducer subset of $\mathrm{T}$ lymphocytes makes them most susceptible to infec-

Correspondence: I.V.D. Weller, B.Sc., M.R.C.P., M.D.

Received: 29 January 1987 tion. These lymphocytes have a pivotal role in the immune response. Their depletion and/or a functional abnormality which results from HIV infection leads not only to a loss of cytotoxic T-cell responses but also a reduction of natural killer cell and monocyte function and B cell abnormalities with polyclonal B cell activation, hypergammaglobulinaemia and impairment of specific antibody responses to new antigens. ${ }^{9}$ In addition, HIV has been shown to infect macrophages, B-lymphocyte cell lines and neuroglial cells. ${ }^{10}$ Seroconversion for anti-HIV occurs typically 4-12 weeks after acute infection, although longer delays in the development of antibody and a persistent antigenaemia in the absence of detectable antibody have been described. The antibodies produced to HIV core and envelope proteins in vivo are a readily detectable marker of exposure, but have only weak in vitro neutralizing activity and therefore coexist with latent infection and productive viral replication. ${ }^{11}$

\section{Acute HIV infection}

Although most acute infections are asymptomatic, a number of syndromes have been described. The commonest is a non-specific viral or glandular-fever type illness occurring one to six weeks after infection. This may present with fever, sweats, malaise, myalgia, arthralgia, sore throat, nausea, headache, diarrhoea, photophobia, a rash and lymphadenopathy. ${ }^{12}$ Seroconversion for anti-HIV does not usually occur until after the symptoms have resolved. An acute meningo-encephalitis and myelopathy have also been described. ${ }^{13,14}$ The encephalitis may present with a prodrome of malaise, fever and personality change, progressing to severe headache, convulsions and various degrees of coma.

(C) The Fellowship of Postgraduate Medicine 1987 


\section{Chronic HIV infection}

The commonest finding in chronic HIV infection is generalized lymphadenopathy. About a third of antiHIV positive patients fulfill the CDC definition of persistent generalized lymphadenopathy (PGL) with nodes of at least $1 \mathrm{~cm}$ in diameter in two extrainguinal sites for 3 months or more. ${ }^{15}$ Many others have lesser degrees of lymphadenopathy. In both categories, the nodes are symmetrical, mobile and non-tender. Referral of every patient with lymphadenopathy for lymph node biopsy is inappropriate. Biopsy is unrewarding in asymptomatic patients revealing only non-specific follicular hyperplasia. If there are constitutional symptoms such as weight loss or fever, or the nodes are markedly asymmetrical, painful or rapidly enlarging or if there is an extranodal mass or coexisting hilar nodes, then biopsy is essential. ${ }^{16}$ This will exclude tumours such as lymphoma or lymphadenopathic Kaposi's sarcoma or an opportunistic infection such as mycobacteria. Tiredness, lethargy, fever and weight loss may occur, without any other identifiable cause. The development of shortness of breath, tachypnoea and even chest discomfort may suggest to both patient and physician the development of a more sinister complication, but anxiety and depression are very common and strong reassurance may improve the situation. Routine investigation may reveal lymphopenia or an immune neutropenia or thrombocytopenia, but these are not usually severe. The lymphopenia is largely due to the reduction in CD4 positive helper cells, but there is no requirement to quantify lymphocyte subsets in routine clinical practice.

Minor opportunistic infection is common. Skin conditions encountered include a range of infections: dermatophytic fungal, viral (recalcitrant warts, herpes simplex and zoster) and bacterial (folliculitis, impetigo, and furunculosis). The spectrum of other skin disorders range from mere dryness to severe seborrhoeic dermatitis. Epstein-Barr virus may be responsible for the recently described hairy leukoplakia on the lateral borders of the tongue. ${ }^{17}$

\section{Risk of progression to AIDS}

The risk of progression to AIDS from published studies ranges from 7 to $30 \%$ over 3 to 4 years; ${ }^{18}$ however, prospective cohort studies only represent a short period of observation from which to draw conclusions about the natural history of HIV infection. Furthermore, some of the early studies were mostly based on patients with PGL which may not be representative of all chronically infected patients. Prognostic markers for disease progression are also being sought from these studies. ${ }^{19}$ Clinical features such as constitutional symptoms, oral candida, leukoplakia, herpes zoster and disappearance of lymph nodes have been recorded. Haematological and immunological abnormalities encountered include cytopenias, particularly progressive depletion of CD4positive lymphocytes; a raised ESR; impairment of in vitro gamma-interferon production, lymphocyte responses to pokeweed mitogen and specific cellular response to HIV. More recently a decline in titre or disappearance of anti-P24 (the antibody to HIV core protein) has been shown to antedate the development of the full-blown syndrome by up to 27 months. ${ }^{20}$ It remains to be seen which of these simple clinical or laboratory abnormalities as opposed to the more sophisticated research tests are the best predictors of progression.

\section{Pneumonia in AIDS}

Pneumocystis carinii pneumonia is the commonest lifethreatening opportunistic infection in patients who progress from chronic HIV infection to AIDS. The presentation is subacute with malaise, fatigue, weight loss, and shortness of breath often developing over several weeks. Typical retrosternal or subcostal chest discomfort associated with increasing shortness of breath, a dry cough and fever finally cause the patient to seek help. The chest X-ray at presentation may be normal or show bilateral, fine infiltrates, typically. perihilar. The arterial oxygen tension is usually depressed, and the carbon monoxide transfer factor, where available, is low and may be the earliest detectable abnormality. The diagnosis is confirmed by induced sputum examination or fibreoptic bronchoscopy with bronchial lavage and transbronchial biopsy, thereby leaving the patient in no doubt as to whether the full syndrome has developed. At the same time other causes of pneumonia or co-existent infection in AIDS patients such as cytomegalovirus (CMV), mycobacteria and other bacteria and fungi may be excluded. ${ }^{21}$

Pyogenic bacterial causes of pneumonia should always be considered, particularly as their presentation may be atypical. In one series, $10 \%$ of episodes of pneumonia in AIDS patients were due to bacteria such as Streptococcus pneumoniae, Haemophilus influenzae, Branhamella and Group B Streptococci. ${ }^{22}$ The radiological appearances may include diffuse infiltrates as well as the more typical focal or lobar patterns. Another cause of diffuse abnormality is lymphocytic interstitial pneumonitis, first described in paediatric AIDS and now increasingly recognized in adults. The cause is still uncertain, but it is certainly associated with HIV infection. 


\section{The gastrointestinal tract}

Any part of the gastrointestinal tract may be affected in AIDS. ${ }^{23}$ Oral candidiasis is very common. Retrosternal discomfort and dysphagia may be due to oesophageal candidiasis, cytomegalovirus (CMV) or herpes simplex virus (HSV) infection. All these have been reported to cause focal or diffuse ulceration and so the diagnosis is best established by endoscopy, biopsy and culture. Diarrhoea and malabsorption may occur without identifiable cause and be associated with partial villous atrophy on biopsy. ${ }^{24}$ Whether HIV is directly responsible for this enteropathy is still under investigation. Cryptosporidium is the commonest of the protozoal infections, which also include Isospora belli and Microsporidia. Cryptosporidium may give rise to persistent or intermittent diarrhoea which ranges from loose stool to torrential diarrhoea with malabsorption, weight loss, fever and abdominal discomfort. The diagnosis is made by finding the cysts in stool by a modified acidfast stain or sucrose concentration and should not be discounted without multiple specimens. CMV and herpes simplex virus (HSV) may cause ulceration in any part of the gut, but most commonly HSV causes mucocutaneous lesions at the upper and lower ends of the gastrointestinal tract, whilst CMV causes a colitis. Atypical mycobacterial infection of the small bowel with Mycobacterium avium intracellulare has been associated with malabsorption and histological appearances similar to Whipple's disease with PAS positive macrophages infiltrating the lamina propria. However, the organisms seen are acid-fast. Mycobacterium tuberculosis infection of the bowel does occur but is less common. Salmonella species may cause diarrhoea, but are more likely in AIDS to cause a fever with bacteraemia.

Hepatitis in AIDS patients most commonly presents with fever, hepatomegaly and abnormal liver function tests, in particular a raised alkaline phosphatase activity. A granulomatous histological picture is the commonest finding and is usually caused by atypical mycobacterial infection. ${ }^{25}$ Cholestasis, acalculous cholecystitis and cholangitis have been recently described with an endoscopic retrograde cholangiographic picture similar to primary sclerosing cholangitis. Cryptosporidia and CMV have both been implicated in this syndrome. ${ }^{26,27}$

\section{Neurological disease}

Neurological disease occurs at all stages of HIV infection. The acute encephalitis has already been described. A chronic encephalopathy is a complication of chronic HIV infection characterized by a subacute dementia with motor dysfunction. ${ }^{28-30}$ Post-mortem studies suggest that up to $95 \%$ of AIDS patients will develop neurological involvement, while clinical assessment identifies at least two thirds. This disorder may predate the diagnosis of AIDS and cases have been reported in whom death occurred due to neurological disease without the patient ever having had any other manifestation of AIDS. Early features are mild and may be described by both patient and friends as a general mental slowing manifest as delayed motor and verbal responses and impaired memory and concentration. Apathy and withdrawal occur, but this must be distinguished from depression which occurs independently. Tremor and ataxia occur commonly. Progression leads to moderate or severe disability in over half of cases in three months and acute exacerbations are common with intercurrent organic illness or psychotropic drugs. Focal signs may develop at any stage but the computed tomographic (CT) scan shows only generalized cerebral atrophy in established cases. The next most common disorder of the central nervous system (CNS) is opportunistic infection with Toxoplasma gondii. ${ }^{31}$ This usually presents with headache, fever and focal signs. The CT scan reveals hypodense lesions with ring contrast enhancement. Double-dose contrast improves the rate of detection, but even then a few cases will only be detected on repeat scanning. The differential diagnosis includes primary CNS lymphoma, fungal, tuberculosis or other bacterial abscess. In patients with meningism and a normal CT scan, the commonest finding is a cryptococcal meningitis, diagnosed by indian ink staining of the cerebrospinal fluid and antigen detection. The spinal cord may be involved by a vascular myelopathy or a transverse myelitis which has been attributed to herpes zoster or simplex, CMV or HIV itself. Two forms of peripheral neuropathy may be encountered; a symmetrical sensorimotor type which may be painful, or a mononeuritis in both of which HIV itself is implicated. Cytomegalovirus retinitis causes progressive visual failure, with a characteristic pattern of exudates and haemorrhages described as 'cottage cheese and jam'.

A pyrexia of unknown origin in an individual at risk may be the only presenting feature and poses a considerable challenge to the skills and resources of physician and microbiologist alike. Mycobacterial infection, typical or atypical, and lymphoma should be high on the list of differential diagnoses.

\section{Tumours}

Much the most common tumour in AIDS patients is Kaposi's sarcoma, presenting as persistent purple skin lesions, flat or raised and usually firm and painless. Palatal lesions are common. Lesions on the face are frequent and particularly distressing. As many as $40 \%$ of patients have small and/or large bowel involvement 
at presentation. Although these lesions are usually clinically silent, they may cause intestinal blood loss, mimic ulcerative colitis, or be associated with a protein losing enteropathy. ${ }^{32,33}$ Primary cerebral lymphoma was recognized early in the epidemic as a feature of AIDS. More recently other non-Hodgkin's lymphomas have been included within the CDC definition. The striking feature is that most $(90 \%$ in one series) have extranodal disease at presentation. ${ }^{34}$ Intestinal involvement is common and often detected only at autopsy. There is a range of histological types but for all types the prognosis is worse than in non-HIV associated cases. There are anecdotal reports of an association between chronic HIV infection and Hodgkin's disease as well. ${ }^{35}$

\section{Treatment of HIV infection}

The CDC have recently proposed a classification scheme for HIV infection related to our present understanding of disease progression. ${ }^{36}$ There are four groups: Group I is acute infection, Group II asymptomatic infection, Group III persistent generalized lymphadenopathy and Group IV other diseases. Subgroups of Group IV are (a) constitutional disease, (b) neurological disease, (c) secondary infectious diseases, (d) secondary cancers and (e) other conditions. The existing definition of AIDS will overlap with subgroups (c) and (d).

At present, the treatment of HIV infection is limited to the treatment of its complications beginning with the psychological morbidity associated with having a positive test result. ${ }^{37}$ Patients should be counselled before as well as after having the test performed. Anxiety and depression are common in the months following the test, but in most cases will respond to repeated reassurance and adequate discussion of any minor symptoms. The opportunistic infections are treated as they would be in other immunosuppressed patients. In general, therapy suppresses rather than eradicates the infection, so relapse is common.

Of the protozoal infections, pneumocystis is the most likely to respond well. Co-trimoxazole remains the treatment of choice. High-dose intravenous therapy $(20 \mathrm{mg} / \mathrm{kg} /$ day of trimethoprim) for 3 weeks is the norm, although once the patient's fever has settled and the shortness of breath and blood gases have improved co-trimoxazole can be given orally. A minor rash during treatment may be ignored, but a very itchy generalized eruption with recurrence of fever, or severe cytopaenia may occur at 7-10 days and requires a change to pentamidine mesylate. This needs to be given by slow intravenous infusion $(2.5 \mathrm{mg} / \mathrm{kg} / \mathrm{day})$ to avoid the persistent pain and sterile abscesses that occur with intramuscular injection. Hypoglycaemia and renal failure may occur during treatment. Alth- ough unproven in controlled studies in AIDS, many physicians would follow a first attack of Pneumocystis carinii pneumonia with some form of prophylaxis such as low dose co-trimoxazole, weekly Fansidar or monthly pentamidine.

Cryptosporidiosis may respond to spiramycin $(4 \mathrm{~g} /$ day $)^{38}$ or a combination of quinine and clindamycin, but reported success is anecdotal and spontaneous remission may occur. There are also anecdotal reports of a response to interleukin- $2 .^{39}$ Isosporiasis responds well to co-trimoxazole ( 8 tablets/day) for 10 days, but relapses occur in $50 \%$ of cases with cessation of treatment. ${ }^{40}$ Cerebral toxoplasmosis responds to sulphadiazine and pyrimethamine, although delayed diagnosis and more severe symptoms are associated with a poor prognosis.

Herpes simplex virus infections respond to oral acyclovir. Prophylactic treatment $(800 \mathrm{mg} /$ day $)$ may be required as severe, frequent recurrences are common. Intravenous acyclovir has been used in other immunocompromised patients for the treatment of herpes zoster infection. ${ }^{41}$ It may also be given orally at a dose of $1.6 \mathrm{~g}$ /day. Ganciclovir (dihydroxypropoxymethyl guanine, DHPG), in uncontrolled studies, has shown promising results in the treatment of CMV retinitis and to a lesser extent pneumonia and colitis, but maintenance treatment is required and even then relapse occurs.

Simple oral candida responds initially to localo nystatin or amphotericin but later may require, and? responds well to, ketoconazole $(200-400 \mathrm{mg} /$ day), which is also the drug of choice in oesophageal candidiasis. Cryptococcal and the rare life-threatening candidal infections required amphotericin and 5fluorocytosine.

Bacterial pneumonias and skin infections will respond to conventional antibiotics. Diarrhoea may respond to metronidazole, even in the absence of recognized pathogens in the stool ${ }^{42}$ Symptomatic treatment with codeine phosphate, loperamide and other drugs can be used, and may be the only effective treatment for cryptosporidiosis. Mycobacterium tuberculosis is treated conventionally. The atypical organisms are resistant to conventional antituberculous therapy. Ansamycin and clofazimine are among the agents being tried.

The median survival from time of diagnosis for patients with Kaposi's sarcoma is substantially better than those with opportunistic infection. In the absence of any evidence for improved survival with early therapy, the first lesions of Kaposi's sarcoma may require no treatment. This is not a reason to delay making or informing the patient of the diagnosis and its implications. Such patients, who are usually free from constitutional symptoms, have longer to come to terms with their illness. Local radiotherapy can be used for treatment of mucocutaneous lesions for 
cosmetic reasons and for larger lesions causing local complications such as those in the oropharynx. Systemic chemotherapy is used in generalized mucocutaneous disease with or without visceral involvement. Various single agent and combined cytotoxic regimes have been used with their many side effects, risk of further immunosuppression and reactivation of latent infection. ${ }^{43}$ Lymphoblastoid and recombinant human alpha-interferons have been used with a few complete remissions. Thirty to forty per cent of patients have partial remissions with this treatment, but severe side effects are common with constitutional symptoms and cytopaenias. At present the best that we should expect with any form of systemic therapy is a temporary, partial remission at considerable cost to the patient in terms of adverse effects and with no evidence yet that the treatment will alter the long-term prognosis. Many centres therefore have a conservative policy for the use of such therapy in most cases of Kaposi's sarcoma.

Most attention is now being focussed on specific inhibitors of the HIV reverse transcriptase. ${ }^{44}$ Inhibition of productive viral replication may allow some recovery of immune function encouraging regression of tumours and eliminating the conditions favouring opportunistic infections. Small, uncontrolled studies with suramin and an antimony compound, HPA 23, showed little benefit and the adverse effects were unacceptable. Phosphonoformate (Foscarnet), a pyrophosphate analogue, has been shown in vitro to inhibit reverse transcriptase but this has not yet been shown in vivo and small controlled studies have so far failed to demonstrate a convincing clinical benefit.

The second generation of reverse transcriptase inhibitors has begun with zidovudine (3'-azido-3'deoxythymidine). Formerly known as azidothymidine (AZT), this compound is showing early promise. It is a thymidine analogue which was found to have considerable in vitro activity against HIV. In phase one studies it was given intravenously at up to $30 \mathrm{mg} / \mathrm{kg} /$ day and was shown also to be orally absorbed and to be able to cross the blood brain barrier. ${ }^{45}$ Approximately 280 patients with past Pneumocystis carinii pneumonia or severe symptomatic chronic HIV infection were subsequently enrolled in a phase two placebo controlled study in 1985 . Only preliminary results have been released but 16 deaths were reported among the 137 patients receiving placebo, while there was one death in the treatment group. In addition there were significantly fewer episodes of opportunistic infection. One small study has shown improvement in neurological disease ${ }^{46}$ The full assessment of the place of this drug in the treatment of AIDS awaits informa- tion about the adverse effects profile as well as its efficacy in other disease groups. The search for other nucleotide analogues continues. The ideal antiviral agent will be specific, orally absorbed, penetrate the CNS and be free from adverse effects. It will then be possible to evaluate its usefulness earlier in the chronic infection. However, the best that we can anticipate is suppression of productive viral replication, and the problem of latently infected cells remains.

\section{HIV infection and the general physician}

The problems posed by HIV infection are not confined to diagnosis and treatment protocols, but begin with the antibody test itself. There is a temptation to treat this test like any other. However, it is recommended that no patient should have their HIV antibody status determined without their consent. Counselling should be provided to prepare a patient before the test and give support and advice when the result is communicated. It is more difficult to maintain confidentiality in a busy casualty or general out-patient setting than it is in a genito-urinary medicine clinic. Nevertheless we have an obligation to strive to maintain it and this should be recognized by all concerned. The consequences for an individual if confidentiality is breached may be devastating. There are many accounts of ostracism, loss of employment and accommodation, and even suicide. The test has been used as a substitute for an adequate history. It is also used on the grounds of infection control when the raising of routine standards of prevention of cross-infection may be more appropriate. HIV has been transmitted to health care workers ${ }^{47}$ but to date only by needlestick exposure and rarely. Only a minority of in-patients need isolation facilities. All hospitals should now have a policy for the management of HIV associated disease. HIV is a problem for the general physician and not merely for the 'AIDS expert'. We have observed a growth of such experts in the five years since 1982 as the number of AIDS cases has risen. As we have come to appreciate the number of individuals with chronic HIV infection, it is clear that the next five years will see a change in the emphasis as most cases should and will be managed by general physicians.

\section{Acknowledgements}

I.V.D. Weller is a Wellcome Trust Senior Lecturer in infectious diseases. 
1. Centers for Disease Control. Revision of the case definition of the Acquired Immunodeficiency Syndrome for national reporting - United States. $M M W R$ 1985, 34: 373-375.

2. Sivak, S.L. \& Wormser, G.P. How common is HTLV-III infection in the United States. $N$ Engl J Med 1985, 313: 1352.

3. Curran, J.W., Meade Morgan, W., Hardy, A.M., Jaffe, H.W., Darrow, W.W. \& Dowdle, W.R. The epidemiology of AIDS: current status and future prospects. Science 1985, 229: 1352-1357.

4. Centers for Disease Control. Heterosexual transmission of human T-lymphotropic virus type III/Lymphadenopathy virus. $M M W R$ 1985, 34: 561 .

5. Biggar, R.J. The AIDS problem in Africa. Lancet 1986, i: 79-82.

6. Wong-Staal, F. \& Gallow, R.C. Human T-lymphotropic retroviruses. Nature 1985, 317: 395-403.

7. Clavel, F., Guetard, D., Brun-Vezinet, F. et al. Isolation of a new human retrovirus from West African patients with AIDS. Science 1986, 233: 343-346.

8. Brucker, G., Brun-Vezinet, F., Rosenheim, M., Rey, M.A., Katlama, C. \& Gentilini, M. HIV-2 infection in two homosexual men in France. Lancet 1987, i: 223.

9. Lawrence, J. The immune system in AIDS. Scientific American 1985, 253: 70-79.

10. Gartner, S., Markovits, P., Markovitz, D.M., Kaplan, M.H., Gallo, R.C. \& Popovic, M. The role of mononuclear phagocytes in HTLV III/LAV infection. Science 1986, 233: $215-219$.

11. Weiss, R.A., Clapham, P.R., Cheingsong-Popov, R. et al. Neutralising antibodies to human T-lymphotropic virus type III. Nature 1985, 316: 69-71.

12. Cooper, D.A., Gold, J., Maclean, P. et al. Acute AIDS retrovirus infection. Lancet 1985 , i: 537-540.

13. Carne, C.A., Tedder, R.S., Smith, A. et al. Acute encephalopathy coincident with seroconversion for antiHTLV III. Lancet 1985, ii: 1206-1208.

14. Denning, D.W., Anderson, J., Rudge, P. \& Smith, H. Acute myelopathy association with primary infection with human immunodeficiency virus. Br Med J 1987, 294: $143-144$.

15. Jaffe, H.W., Darrow, W.W., Edenberg, D.F. et al. The acquired immunodeficiency syndrome in a cohort of homosexual men. A six year follow up study. Ann Int Med 1985, 103: 210-214.

16. Rashleigh-Belcher, H.J.C., Carne, C.A., Weller, I.V.D., Smith, A.M. \& Russell, R.G.G. Surgical biopsy for persistent generalised lymphadenopathy. Br J Surg 1986, 73: $183-185$.

17. Greenspan, J.S., Greenspan, D., Lennette, E.T. et al. Replication of Epstein-Barr virus within the epithelial cells of oral "hairy" leukoplakia, an AIDS-associated lesion. $N$ Engl J Med 1985, 313: 1564-1571.

18. Goedert, J.J., Biggar, R.T., Weiss, S.H. et al. Three year incidence of AIDS in five cohorts of HTLV III infected risk group members. Science 1986, 231: 992-995.

19. Anonymous. Who will get AIDS. Lancet 1986, ii: 953954.

20. Weber, J.N., Clapham, P.R., Weiss, R.A. et al. Human immunodeficiency virus infection in two cohorts of homosexual men: neutralising sera and association of anti-gag antibody with prognosis. Lancet 1987, i: 119122.

21. Murray, J.F., Felton, C.P., Garay, S.M. et al. Pulmonary complications of the acquired immunodeficiency syndrome. N Engl J Med 1984, 310: 1682-1688.

22. Polsky, B., Gold, J.W.M., Whimbey, E. et al. Bacterial pneumonia in patients with the acquired immunodeficiency syndrome. Ann Int Med 1986, 104: 38-41.

23. Weller, I.V.D. AIDS and the gut. Scand J Gastroenterol 1984, 20: (Suppl 114): 77-89.

24. Kotler, D.P., Gaetz, H.P., Lange, M., Klein, E.B. \& Holt, P.R. Enteropathy associated with the acquired immunodeficiency syndrome. Ann Int Med 1984, 104: 421-428.

25. Gordon, S.C., Rajender Reddy, K., Gould, E.E. et al. The spectrum of liver disease in the acquired immunodeficiency syndrome. $J$ Hepatol 1986, 2: 475-484.

26. Kavin, H., Jonas, R.B., Chowdhury, L. \& Kabins, S. Acalculous cholecystitis and cytomegalovirus infection in the acquired immunodeficiency syndrome. Ann Int Med 1986, 104: 53-64.

27. Margolis, S.J., Honig, C.L., Soave, R., Govoni, A.F., Mowadian, J.A. \& Jacobson, I.M. Biliary tract obstruction in the acquired immunodeficiency syndrome. Ann Int Med 1986, 105: 207-210.

28. Price, R.W., Navia, B.A., Cho, E-S. AIDS encephalopathy. Neurol Clin 1986, 4: 285-301.

29. Navia, B.A., Jordan, B.D. \& Price, R.W. The AIDS dementia complex: I. Clinical features. Ann Neurol 1986 19: $517-524$.

30. Navia, B.A., Cho, E-S, Petito, C.K. \& Price, R.W. The AIDS dementia complex: II. Neuropathology. Ann Neurol 1986, 19: 525-535.

31. Navia, B.A., Petito, C.K., Gold, J.W.M., Cho, E-S., Jordan, B.D. \& Price, R.W. Cerebral toxoplasmosis complicating the acquired immune deficiency syndrome: clinical and neuropathological findings in 27 patients. Ann Neurol 1986, 19: 224-238.

32. Weber, J.N., Carmichael, D.J., Boylston, A. et al. Kaposi's sarcoma of the bowel - presenting as apparent ulcerative colitis. Gut 1985, 26: 295-300.

33. Friedman, S.L., Wright, T.L. \& Altman, D.F. Gastrointestinal Kaposi's sarcoma in patients with acquired immunodeficiency syndrome. Gastroenterology 1985, 89: 162-168.

34. Ziegler, J.L., Beckstead, J.H., Voldberding, P. et al. Non-Hodgkin's lymphoma in 90 homosexual men: relation to generalised lymphadenopathy and the acquired immunodeficiency syndrome. $N$ Engl J Med 1984, 311: 565-570.

35. Baer, D.M., Anderson, E.T. \& Wilkinson, L.S. Acquired immune deficiency syndrome in homosexual men with Hodgkin's disease: three case reports. Am J Med 1986, 80: $738-40$.

36. Centers for Disease Control. Classification system for human T-lymphotropic virus type III/lymphadenopathy associated virus infections. $M M W R$ 1986, 35: 334-339.

37. Miller, D. \& Green, J. Psychological support and counselling for patients with acquired immune deficiency syndrome (AIDS). Genitourin Med 1985, 61: 273-278.

38. Portnoy, D., Whiteside, M.F., Buckley III, E., Macleod, C.L. Treatment of intestinal cryptosporidiosis with 
spiramycin. Ann Int Med 1984, 101: 202-204.

39. Kern, P., Toy, J. \& Dietrich, M. Preliminary clinical observations with recombinant Interleukin-2 in patients with AIDS or LAS. Blut 1985, 50: 1-6.

40. Dettovitz, J.A., Pape, J.M., Boncy, M. \& Johnson, W.D. $\mathrm{Jr}$. Clinical manifestations and therapy of isospora belli infection in patients with the acquired immunodeficiency syndrome. $N$ Engl J Med 1986, 315: 87-90.

41. Shepp, D.H., Dandliker, P.S. \& Meyers, J.D. Treatment of varicella-zoster virus infection in severely immunocompromised patients. A randomised comparison of acyclovir and vidarabine. $N$ Engl J Med 1986, 314: 208 212.

42. Rolston, K.V.I., Hoy, J. \& Mansell, P.W.A. Diarrhoea caused by "non pathogenic amoebae" in patients with AIDS. $N$ Engl J Med 1986, 315: 192.

43. Krigel, R.L., \& Friedman-Kien, A.E. Kaposi's sarcoma in AIDS. In De Vita Jr., V.T., Hellman, S. \& Rosenberg, S.A. (eds) AIDS Etiology, Diagnosis, Treatment and Prevention. J.B. Lippincot, Philadelphia, 1985, pp185-211.

44. Gupta, S. \& Gottlieb, M.S. Treatment of the acquired immune deficiency syndrome. J Clin Immunol 1986, 6: 183-193.

45. Yarchoan, R., Klecker, R.W., Weinhold, K.J. et al. Administration of $3^{\prime}$-azido-3'deoxythymidine; an inhibitor of HLTV III/LAV replication, to patients with AIDS or AIDS-related complex. Lancet 1986, i: 575580.

46. Yarchoan, R., Berg, G. \& Brouwers, P. Response of human immunodeficiency virus associated neurological disease to $3^{\prime}$-azido-3'deoxythymidine. Lancet 1987 , i: $132-135$

47. Geddes, A.M. Risk of AIDS to health care workers. $\mathrm{Br}$ Med J 1986, 292: 711-712. 